\title{
We Know Now: Mavridis Atrophy Is Associated with Cognitive Impairment in Parkinson Disease
}

l: read the recent article by Mak et al $^{1}$ regarding the association of subcortical atrophy with cognitive impairment in Parkinson disease (PD), with great interest. The authors assessed cortical thickness and subcortical volumes in patients with PD without dementia and evaluated their associations with cognitive dysfunction. They found that patients with PD and mild cognitive impairment demonstrated reduced volumes of the thalamus and nucleus accumbens (NA) and significant associations for the NA and putamen with performances on the attention/working memory domains and NA and language domains. ${ }^{1}$ The purpose of this communication is to comment on the phenomenon of NA atrophy in $\mathrm{PD}$ and its correlation with cognitive symptoms.

In the clinical study of Mak et al, ${ }^{1}$ the total volume of the NA was significantly correlated with a range of cognitive variables, including overall scores of attention/working memory and language. They also found that a trend toward the associations between NA and global cognition and executive function performance on attention/working memory was associated with reduced volumes of the NA and putamen. Additionally, the NA was also significantly correlated with performance in the language domain. The authors suggested, as possible explanation for their findings, the previous evidence linking the NA to memory and learning processes. ${ }^{1}$

Although the involvement of subcortical deep gray matter and cortical thinning associated with mild PD remains poorly understood, ${ }^{1}$ we currently know that atrophy of the human NA in PD, called Mavridis atrophy (MA), begins in early-stage PD and is correlated with psychiatric symptoms that occur in $\mathrm{PD}$, mainly apathy and impulsive behavior. ${ }^{2}$ Two recent clinical studies have also associated the phenomenon of MA with cognitive PD symptoms. ${ }^{2-4}$ More specifically, O'Callaghan et $\mathrm{al}^{3}$ found reduced learning rates in patients with $\mathrm{PD}$ without dementia relative to

http://dx.doi.org/10.3174/ajnr.A4315 controls and that this learning impairment was directly related to gray matter loss in discrete frontostriatal regions, including the NA. Hanganu et $\mathrm{al}^{4}$ found a significant decrease in the NA volume in patients with PD and mild cognitive impairment. Therefore, the study of Mak et al ${ }^{1}$ is the third clinical study to confirm the MA association with cognitive symptoms in PD.

Finally, Mak et $\mathrm{al}^{1}$ proposed that nuclei such as the NA may serve as potential biomarkers for PD-mild cognitive impairment. In agreement with their advice, previous authors have suggested that it is time to evaluate MA (as an imaging finding) as a risk factor for the expression of specific PD symptoms. The evaluation should emphasize those symptoms that we already know are related to MA, namely psychiatric and cognitive symptoms. It is also time to evaluate MA as a risk factor (prognostic factor) for the severity of the disease. ${ }^{2}$ Further research is definitely needed, and PD seems ready to reveal some of its well-kept secrets.

\section{REFERENCES}

1. Mak E, Bergsland N, Dwyer MG, et al. Subcortical atrophy is associated with cognitive impairment in mild Parkinson disease: a combined investigation of volumetric changes, cortical thickness, and vertex-based shape analysis. AJNR Am J Neuroradiol 2014;35: 2257-64

2. Mavridis IN. Mavridis' atrophy in Parkinson's disease: "the peak of the iceberg." J Transl Intern Med 2014;2:124-26

3. O'Callaghan C, Moustafa AA, de Wit S, et al. Fronto-striatal gray matter contributions to discrimination learning in Parkinson's disease. Front Comput Neurosci 2013;7:180

4. Hanganu A, Bedetti C, Degroot $C$, et al. Mild cognitive impairment is linked with faster rate of cortical thinning in patients with Parkinson's disease longitudinally. Brain 2014;137:1120-29

I.N. Mavridis

Department of Neurosurgery “K.A.T.-National Rehabilitation Center" General Hospital of Attica Athens, Greece 